\section{THE HRB NATIONAL DRUGS LIBRARY: BREAKING BOUNDARIES BY BRIDGING AND BONDING}

Mary Dunne. Health Research Board, Dublin, Ireland

10.1136/bmjebm-2019-EBMLive.89

Objectives Librarians and information professionals in healthcare are perhaps best known for providing access to resources, information literacy training and literature searching. However, librarianship is evolving, and our roles are expanding.

For librarians in the HRB National Drugs Library, providing full and free access to research on substance use remains at the core of our work. Having this evidence in one nationally accessible place was our founding purpose. Yet we know that simply providing access, or the passive dissemination of research, is unlikely to have a significant impact on policy and practice. Therefore, we have redefined our purpose as facilitators of evidence-based decision making and are implementing new interventions that have evidence of impact.

Method Evidence from research literature is helping us achieve our goals. For example, an important finding for us regarding interventions to increase research use was the need for significant social interaction with stakeholders. Although we have engaged with library users and developed active communication and dissemination techniques, other interventions such as building social and professional evidence use norms, consensus-building, and network analysis typically go beyond traditional library services.

Therefore, to understand how we may implement actions in this area we are using evidence related to social networks and interaction. For example, social capital provides access to resources embedded in social relationships. Two forms, each facilitating different beneficial behaviours, are bonding social capital (facilitating shared customs, norms, vision and trust in groups and communities with similar identities) and bridging social capital (providing access to novel resources and information across social relationships with diverse identifies).

Results It seems natural for librarians to develop bridging social capital, which focuses on the circulation of resources like information through relationships with brokerage or weak ties. This way we can connect with individuals and groups across stakeholders including educators, researchers, students, practitioners, and policymakers. We are now also recognizing the benefits of the stronger ties we have made with those who share our aim to apply evidence-based decision making and are using these connections to bring people together. We have begun the process by targeting key stakeholder groups, enabling them to identify relevant research gaps, and commissioning reviews using research teams with community representatives to guide their direction. Our latest initiative involves a cross-disciplinary community of practice to focus on an area of addiction treatment.

Conclusions Much of what we have done over the years has been based on experience. It made sense to us to build relationships with library stakeholders, include them in reviews, and join cross-organisational committees. It also makes sense for us to understand and share the evidence underpinning why we do what we do and how we aim to achieve our goals effectively. We would, therefore, like to present the more novel and social-orientated aspects of our work. We welcome feedback from Evidence Live delegates on our role as librarians and information professionals, so we can progressively enable the systematic use of evidence in policy and practice.

\section{A PRAGMATIC TRIAL OF BLENDED LEARNING VERSUS ONLINE LEARNING FOR CLINICALLY INTEGRATING EVIDENCE BASED MEDICINE TEACHING IN AN UNDERGRADUATE MEDICAL SCHOOL}

${ }^{1}$ Bharathy Kumaravel, ${ }^{2}$ Dragan $\| l i c,{ }^{1}$ Claire Stewart, ${ }^{1}$ Claire Stocker, ${ }^{1}$ Peter Thomas. ${ }^{1}$ University of Buckingham Medical School, Buckingham, UK; ${ }^{2}$ Monash University, Melbourne, Australia

\subsection{6/bmjebm-2019-EBMLive.90}

Objectives Multifaceted, clinically integrated teaching of Evidence Based Medicine (EBM) with assessments are more likely to improve students' knowledge, skills and attitudes. Currently, there is limited evidence on what are the minimum components of the multifaceted interventions or on the most effective models of clinically integrating EBM teaching. The aim of this study is to evaluate the effectiveness of two different models of clinically integrating EBM teaching - blended or online learning.

Method EBM is a progressive longitudinal theme in the University of Buckingham Medical School, where students are taught to ask, acquire and appraise evidence in years one and two (phase I). In years three and four (phase II), students are asked to apply EBM in clinical practice and reflect on their experience. All students received the same educational intervention for phase I. Students in phase II are placed at one of two hospitals, where they received either blended learning (a combination of lectures, facilitated small group discussions) or online learning (recorded lectures and online learning resources).

Learning outcomes assessed included students' EBM knowledge, skills and behaviour using the validated assessment toolAssessing Competency in Evidence Based Medicine (ACE). In addition, students were asked to complete educational prescriptions (EP) - where they developed a question from a clinical scenario, searched and appraised evidence and applied it to the clinical decision.

Results Education was delivered to 65 students, from which 46 students completed the ACE test (32 blended/14 online). There were $31 \mathrm{EP}$ submissions ( 23 blended/8 online learning). Students' performances in both the ACE test and EP were better in the blended learning model compared to online learning. The mean difference for performances in ACE and EP were 1.02 (one tailed $\mathrm{p}$ value $<0.05)$ and $2.29(\mathrm{p}<0.05)$ respectively.

This study was a pragmatic trial; hence it was not possible to either randomise or blind individual students to the interventions. The blended learning approach was resource intensive and needed a lot of planning and commitment. It was feasible in this small teaching hospital with a small cohort of students- whether it is applicable in larger teaching hospitals with bigger cohorts of students is uncertain.

Conclusions Our study demonstrates that it was feasible to offer both models of clinically integrated EBM teaching. Blended learning model is more effective than online learning for clinically integrating EBM teaching as demonstrated by the medical students' competency in EBM knowledge, skills and behaviour using validated assessment tools such as the ACE and EP. In designing teaching methods for clinically integrating EBM, educators need to balance resource implications, students' preferences and impact on learning outcomes. Further research into the minimum components needed for multifaceted interventions and the most efficient models of clinically integrating EBM teaching is needed. 